Musées, Patrimoine et Culture scientifiques et techniques

$184 \mid 2019$

juillet-août 2019

\title{
Quelques principes clefs des expositions de Georges Henri Rivière
}

Jean-Claude Duclos

\section{OpenEdition}

\section{Journals}

Édition électronique

URL : http://journals.openedition.org/ocim/2896

DOI : 10.4000/ocim.2896

ISSN : 2108-646X

Éditeur

OCIM

Édition imprimée

Date de publication : 1 juillet 2019

Pagination : 18-21

ISSN : 0994-1908

Référence électronique

Jean-Claude Duclos, «Quelques principes clefs des expositions de Georges Henri Rivière », La Lettre de l'OCIM [En ligne], 184 | 2019, mis en ligne le 01 juillet 2020, consulté le 24 janvier 2021. URL : http:// journals.openedition.org/ocim/2896 ; DOI : https://doi.org/10.4000/ocim.2896

Ce document a été généré automatiquement le 24 janvier 2021.

Tous droits réservés 


\section{Quelques principes clefs des expositions de Georges Henri Rivière}

Jean-Claude Duclos

Georges Henri Rivière lors d'une collecte en Sologne en 1938

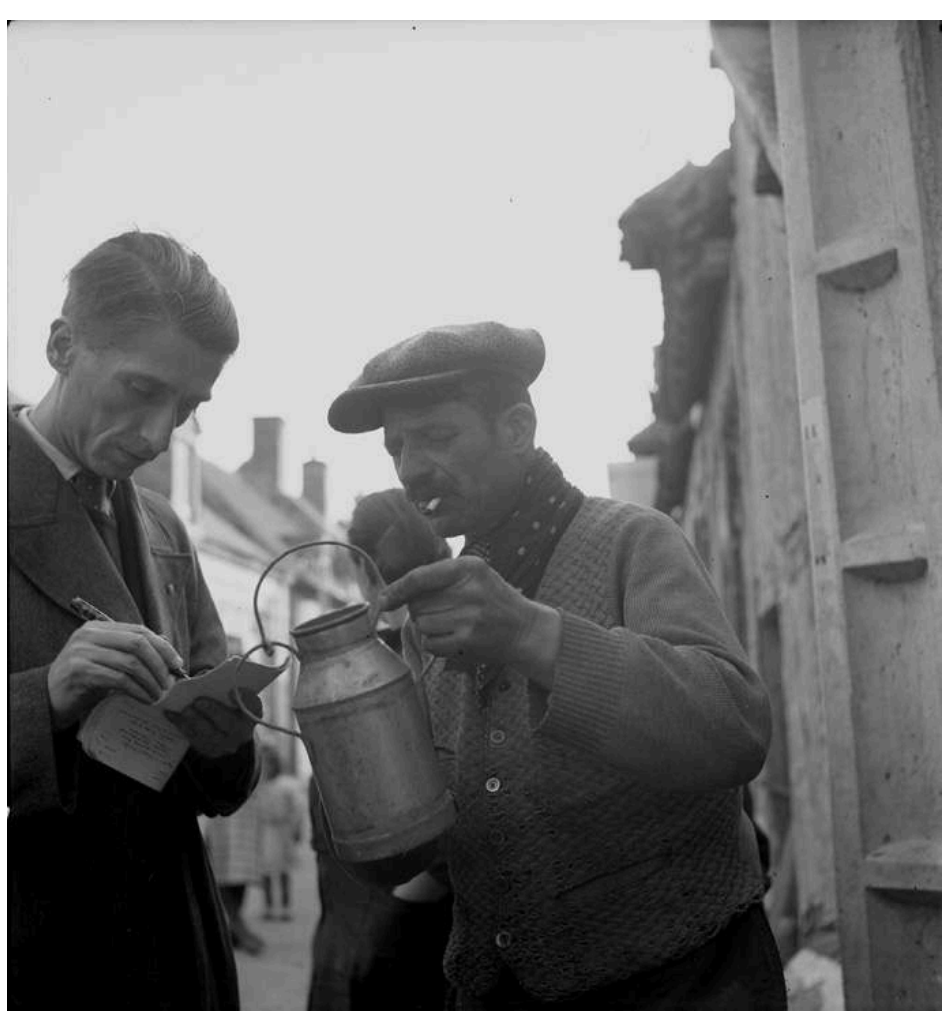

(c) Mucem/Louis Dumont 
1 Qui apprend la muséographie à ses côtés, au début des années 1970, en observant ce qu'il réalise au Musée national des Arts et Traditions populaires (MNATP) et en suivant ses cours de muséologie - c'est mon cas -, en retient des principes qui, bien souvent, sont devenus des règles. Or, attentif à préserver en tout la possibilité d'évoluer et d'innover, GHR préfère les recommandations aux principes. Là réside l'originalité de son enseignement alliant à sa sensibilité d'artiste et aux observations qu'il multiplie sa vie durant dans les musées du monde, le fruit des expérimentations qu'il ne cesse de faire, un demi-siècle durant, de la rénovation du musée d'Ethnographie du Trocadéro aux applications du concept d'écomusée. D'où l'absence d'un manuel de muséographie qu'il se refusera à faire " car ça changera ", répète-t-il, et la difficulté, après sa disparition, lors de la publication de son cours, d'en recomposer un ensemble complet et cohérent. S'il se base sur son expérience, il s'en réfère en effet très souvent à celles des autres (New York, San Francisco...) et jamais ne se dit l'auteur de ce qu'il préconise. Distinguant les présentations scientifiques des présentations culturelles, il ne cesse aussi de répéter que ces dernières ont d'abord pour objectif de répondre aux attentes et aux questionnements du public. C'est d'elles qu'il est question au travers des quelques principes énumérés ci-après.

\section{Le discours}

2 Il s'agit, selon ses propres termes, de "l'organisation idéologique du message à transmettre ", soit d'une première étape qui, conduite en étroite relation avec l'expert ou les experts des disciplines concernées, permet d'établir la base scientifique à partir de laquelle formuler le message de l'exposition. «Liquidation de ces rideaux de fer que les routines muséographiques font tomber entre les disciplines scientifiques " s'exclame-t-il en 1966 pour insister sur la nécessité de l'interdisciplinarité. Cette étape est celle du " programme » qu'il qualifie souvent de " scientifique ", celle de l'état des lieux des connaissances qu'exige le traitement du thème de l'exposition et de l'écriture du discours que l'on souhaite tenir au futur visiteur.

\section{Le parcours}

C'est en passant du " programme » au " projet » qu'est défini le parcours, soit, dans un espace donné, la mise au point d'un cheminement et de ses " distances ", selon la structure de "l'histoire à raconter ", ses parties, son rythme, ses pauses... GHR fait alors souvent référence à la musique où, à propos des pauses, dit-il, « le silence est déjà du son ». Préconisant fréquemment l'approche chronologique et " périodisée », il y introduit "l'alternance de la diachronie et de la synchronie ", soit à la suite d'une partie où le visiteur doit éprouver la sensation du temps qui s'écoule, une autre où le temps s'arrête pour lui montrer ce qui se passe à l'instant $\mathrm{T}$. Le dialogue, au cours de cette étape, reste constant entre les chercheurs et le « muséographe-designer ».

\section{La neutralisation de l'environnement}

4 Partisan d'une présentation épurée des objets où « l'écran d'aucun appareil décoratif indiscret ne doit s'interposer entre eux et le public », il insiste : « La sobriété doit 
régner, la présentation la plus simple est celle qui, à force d'être utile et belle, se fait oublier ». Pour ne plus favoriser que l'objet et concentrer sur lui le regard du visiteur, GHR préconise des fonds sombres où non éclairés afin que la lumière irradie de l'objet lui-même. Il montre quelle grande part occupe la maîtrise de l'éclairage artificiel, dans la communication des objets exposés. D'où la critique lui sera faite de réaliser des « musées noirs ».

\section{La contextualisation}

5 Montrer l'objet doit aussi permettre de « comprendre les sens multiples dont il témoigne ». Son expérience de l'ethnographie, tant au cours des grandes missions du musée de l'Homme des années 1930, que de celles qu'il dirige à travers la France depuis le MNATP, lui font très tôt reconnaître les capacités de "l'habitant-expert ». Même si des investigations, des recoupements et des mises en forme sont encore nécessaires, les informations recueillies doivent être transmises. Ce sont elles qui confèrent à l'objet sa valeur et son intérêt et doivent accompagner sa monstration. D'où le recours au texte, à l'image fixe ou animée, à la carte, au modèle... en prenant soin qu'ils ne nuisent en rien à la présentation de l'objet. Ainsi, des textes « primaires » (qui introduisent les grandes parties), « secondaires » (qui en commentent les sous-parties) et « tertiaires » (ceux des notices), seuls les derniers sont généralement disposés dans la vitrine, au bas et les autres, à l'extérieur. D'où, aussi, la " mise en situation » de l'objet, dans sa position d'utilisation. L'importance qu'il accorde à la contextualisation se manifeste aussi dans les ensembles et notamment dans ceux de la galerie culturelle du MNATP ( Du berceau à la tombe ", " Du blé au pain ", "La transhumance »...) et culmine dans les " unités écologiques " où, avec une précision d'archéologue, l'intégralité des objets " en situation » et de leur environnement physique et sonore, est restituée. Partout doit être maitrisée " la tension entre le désir de montrer et celui de dire ». Si la question de la contextualisation est ici plus largement commentée, c'est parce qu'elle occupe chez GHR une place de plus en plus importante au cours de son cheminement, des années 1920, lors de la visite des musées américains et des musées de plein-air nord-européens, aux années 1960-1970. La création des parcs naturels régionaux et des écomusées, dans le contexte social et politique d'alors, lui en offre l'opportunité. En 1957, il écrit déjà en concevant le programme du musée de Bretagne : « Deux thèmes vers lesquels doivent tendre les présentations muséales s'imposent alors : le temps et l'espace autour d'un territoire donné, les rapports de l'homme et de la nature ». Cette volonté d'une appréhension muséale globale de l'environnement naturel et humain, mise en application dès 1937, se retrouvera dans les versions successives de sa définition évolutive de l'écomusée. 
La Camargue avant et depuis l'apparition de l'homme, musée camarguais, 1979

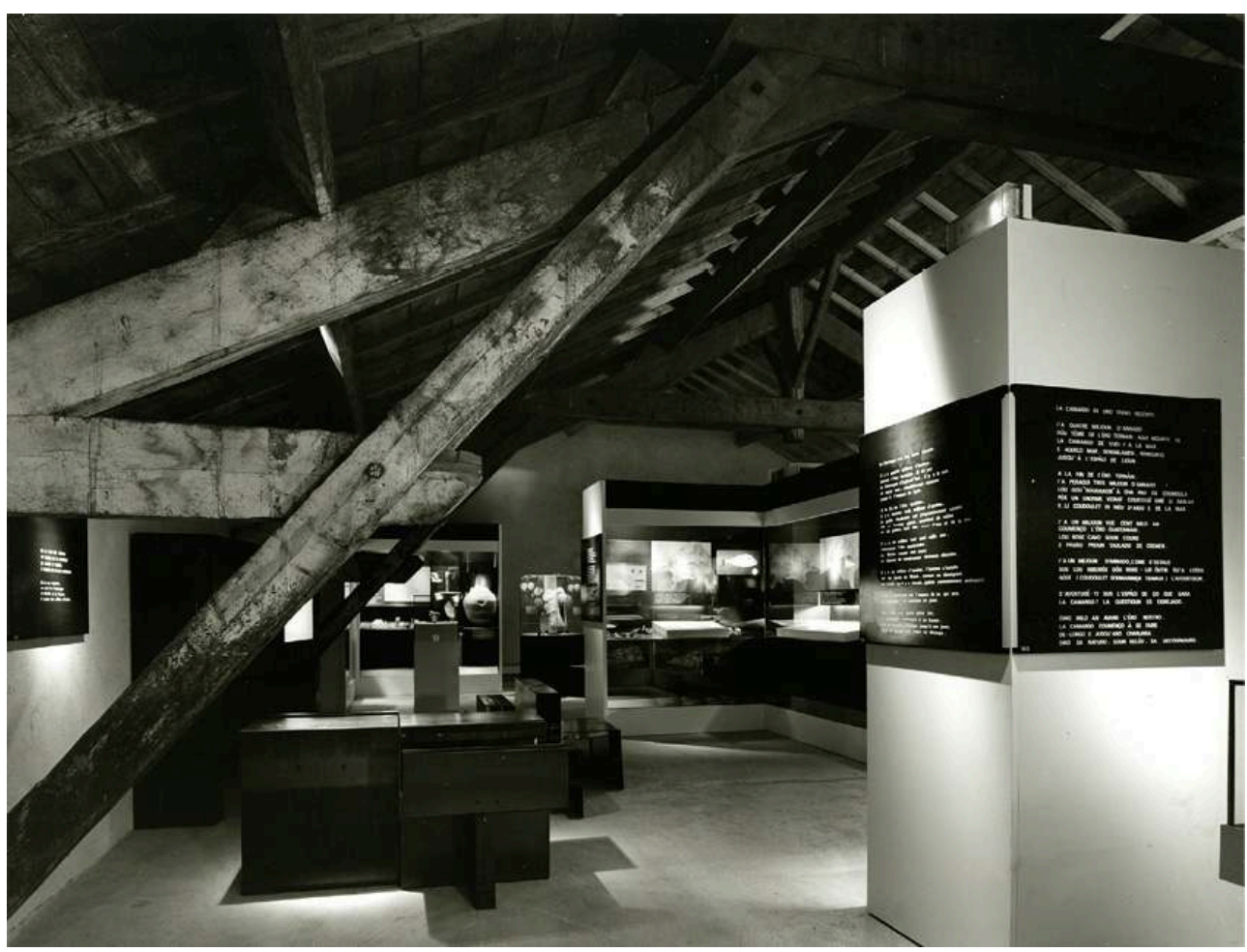

(c) Jean Dieuzaide, coll. Parc naturel régional de Camargue

\section{L'esthétique et « la délectation »}

6 Si « le musicien de vocation, ethnologue et muséologue de fortune » qu'il se dit être et, nourri de toutes les avant-gardes de son temps, l'artiste qu'il est, y attache autant d'importance c'est aussi pour « la délectation » que doit retirer le visiteur de ce qui lui est donné à voir. Il a d'ailleurs réussi à faire admettre ce mot par l'Icom dans la définition du musée.

\section{La participation}

7 Déjà évoquée ci-dessus avec la reconnaissance de "l'habitant-expert ", et pratiquée de longue date par GHR avec le monde associatif, cette question revient en force lors de la phase préparatoire de la création des parcs naturels régionaux (Lurs, 1966) où GHR va jusqu'à se déclarer partisan de l'autogestion, puis lors de la $9^{\mathrm{e}}$ conférence de l'Icom, intitulée Le musée au service des hommes, aujourd'hui et demain (Paris, Dijon, Grenoble, 1971), fondatrice de l'éco-muséologie. Là apparaît le mot écomusée et sa première définition, cosignée par Hugues de Varine et GHR. Dans la conférence qu'il donne à Grenoble en 1978 sur l'écomusée, GHR déclare qu'il faut solliciter la participation " par tous les moyens possibles " mais, déjà conscient de ses limites, prend soin d'ajouter « et ne pas décevoir les élus ». 
Complément du « musée du temps » qu'est le musée camarguais, «l'espace » sur le Sentier de découverte des paysages du mas.

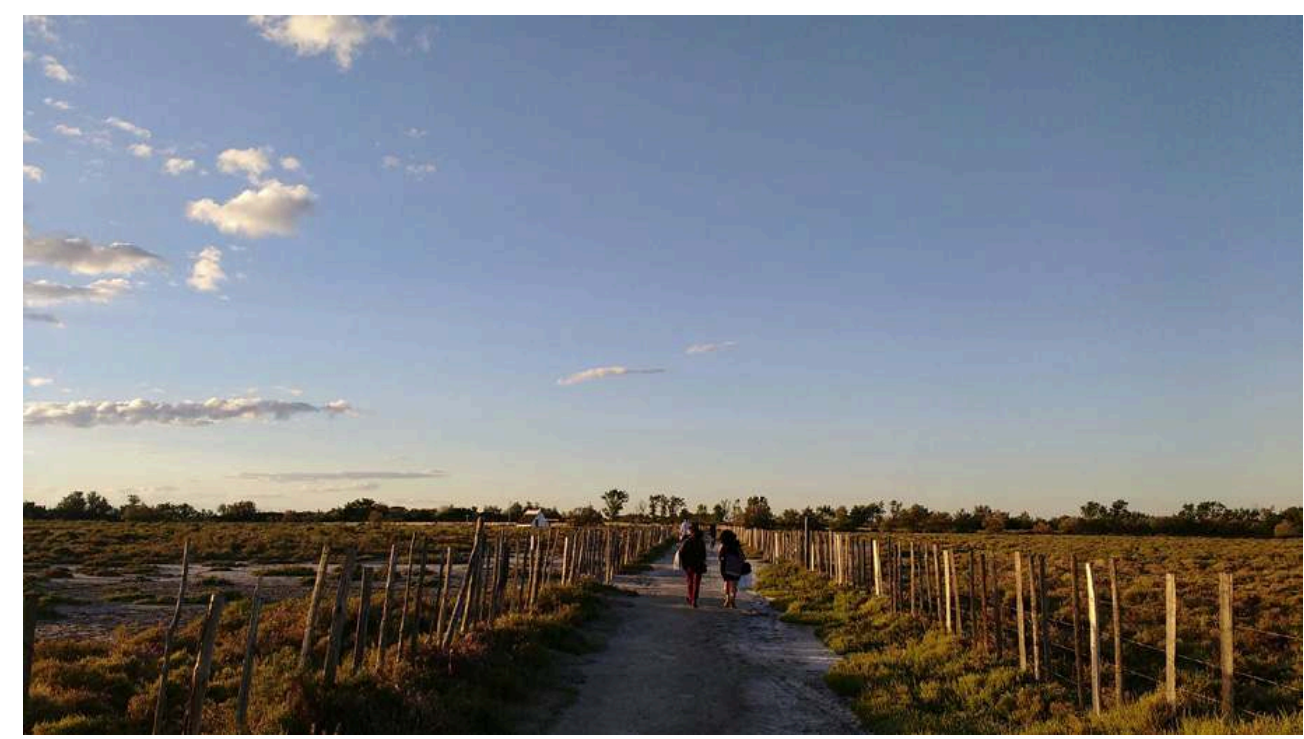

(c) Estelle Rouquette, coll. Parc naturel régional de Camargue

\section{L'évolutivité}

Même lorsqu'il s'agit de " présentation permanente ", GHR s'empresse d'ajouter « évolutive ». Son principal collaborateur au MNATP, André Desvallées, rappelle qu'il estime à dix ans, en 1975, la durée de vie de la Galerie culturelle du MNATP et dit qu'il faudra la reconcevoir, passé ce délai. Or elle sera restée en place durant trente ans. Cette précaution lui fait conseiller des aménagements muséographiques mobiles ou démontables, indépendants quoi qu'il en soit de la structure générale du bâtiment du musée.

9 Tels sont, extraits de l'enseignement de GHR et très sommairement évoqués, les grands principes de la muséographie qui, quant à moi, m'ont permis de réaliser deux musées et une cinquantaine d'expositions. Le premier d'entre eux, le musée camarguais (appelé aujourd'hui le musée de la Camargue), réalisé entre 1975 et 1979, résulte d'une stricte application de ces principes. Ils ont été transmis par la suite durant quelques décennies et le sont encore peut-être aujourd'hui aux stagiaires que nous avons accueillis dans les musées où nous avons travaillé, comme aux étudiants que nous avons contribué à former.

Bien sûr, ces principes évoluent et GHR qui souhaitait cette évolution en serait certainement le premier intéressé. Je pense par exemple à la participation qui est désormais sollicitée dès la phase du discours, lors de la formulation du programme, ou encore à la contextualisation qui, avec les ouvertures de la scénographie, permet d'en inventer de nouvelles formes. L'un ou l'autre de ces points et des questions qu'il soulève dans le processus de mise en exposition serait-il aujourd'hui périmé ? Je ne le pense pas et veux dire à nouveau, avec d'autres plus nombreux qu'on ne croit, combien l'enseignement de GHR nous demeure utile et nécessaire. 


\section{BIBLIOGRAPHIE}

Desvallées, A. L'expologie de Georges Henri Rivière, in Christophe, J., Boëll, D.-M. et Meyran, R. (dir.) Du folklore à l'ethnologie. Édition de la Maison des Sciences de l'Homme, 2009, pp. 277-281.

Weis, H (dir), La muséologie selon Georges Henri Rivière. Cours de muséologie, textes et témoignages. Paris : Dunod, 1989, 402 p.

\section{RÉSUMÉS}

Cet article est extrait de dossier «L'exposition ethnographique selon Georges Henri Rivière ». Ce dossier est l'occasion de reparler d'une question essentielle : en quoi les multiples expositions qu'a conçues et coordonnées Georges Henri Rivière ont-elles renouvelé le mode de collecte et d'exhibition des collections ethnographiques? Au point de faire de la muséologie selon GHR un tournant dans l'écriture du discours expographique et une référence connue dans le monde entier. Il s'agit de rappeler, de façon claire et concise, quels ont été les principes clefs de son inventivité et de sa créativité expographique à travers quelques exemples. Marie-Charlotte Calafat et Germain Viatte reviennent sur les restitutions fidèles réalisées pour l'exposition du Mucem. Jean-Claude Duclos évoque l'exposition initiale, fidèle à l'enseignement de GHR, qu'il a conçue en Camargue. Daniel Jacobi prend exemple du musée du vin de Bourgogne à Beaune pour souligner les qualités esthétiques de cette muséologie. Enfin, Serge Chaumier replace la période Rivière dans l'évolution des expositions dans les musées dits de société.

\section{INDEX}

Mots-clés : Georges Henri Rivière, muséographie, exposition

\section{AUTEUR}

\section{JEAN-CLAUDE DUCLOS}

Ancien directeur du musée dauphinois

jc.duclos@orange.fr 